\title{
Index autorum ad Vol. 166
}

Confecit: C. Loeb-Schürch $(B)=$ Book reviews - Buchbesprechungen - Livres nouveaux

Agarwal, I. P., 188 Albert, M., 236 (B) Alexandridis, E., 236 (B) Arkin, W., 436

Divisová, G., 251 Djakonowa, S. W., 88 Dobrescu, Gioconda, 161 Ducrey, N., 48

Balestrazzi, E., 172, 289 Barishak, R., 301 Bellone, G., 156 Ben-Tovim, T., 81, 353 Bertrand, J. J., 61 Bijlenga, R, 180 Boniuk, I., 234 (B) Bonomi, L., 311 Brancato, R., 105 Busch, I., 144 Cambie, E., 222 Casey, T. A., 233 (B) Cernea, P., 161 Chaurasia, B. D., 188 Cioli, S., 226 Clark, B. A. J., 194 Collignon-Brach, J., 110 Coppieters, R., 401

Daele, R. van, 401

Daicker, B., 180, 233 (B), 460

Delmarcelle, Y., 110

Denden, A., 94

DErmo, F., v. Ermo, F. d'

Dietze, U., 76

Ermo, F. d', 311 Estupinan-Fazio, C, 210

Faussone, Pellegrini, M. S.,

105 Feher, J., 222 Ferraz, O. S. B., 401 Francois, J., 210, 222, 372, 401

Gallenga, P. E., 156 Gdal-On, M., 321 Genovesi, E., 399 Gernet, H., 120 Grail, Y., 61 Grignolo, F., 199

Hammami, H., 16, 241 Hanselmayer, H., 175 Heckmann, K., 36 Hellner, K. A., 327 Hosni, F. A., 265 Hugonnier, R., 233 (B)

Jain, I. S., 306 Jarulin, G. R., 88 Johnston, G. P., 234 (B)

Index autorum ad Vol. 1(

Karel, I., 251 Khare, O. N., 188 Klemetti, Anneli, 441 Kurz, O., 293

Lang, J., 234 (B) Litricin, Olga, 473 Littann, K. E, 94 Lowe, R. F., 194 Lukassek, B., 144

Machtey, L., 293 Manor, Riri Sylvia, 10 Massin, M., 235 (B) Méhes, K., 360 Meyer, E., 321

Netter, G., 450

Okun, E., 234 (B) Ostholt, H., 120

Peleska, M., 251 Piérard, L, 401 Przondka, L., 436

Rickers, L, 327 Rivara, A., 199 Romano, A., 81, 301, 353

Romem, M., 71

Sachs, W., 10 Schlote, W., 477 (B) Schmöger, E., 144 Scouras, L, 450 Scuderi, G., 172, 349

Sebestyén, J., 360 Segal, E., 81,353 Singh, Kapalmit, 306 Slatt, 477 (B) Stein, 477 (B) Stein, R., 301 Streiff, E. B., 16,241

Tarkkanen, A., 441 Theodossiadis, G., 1 Tilgner, S., 76

V. Vand Daele

$\mathrm{R}$.

v. Daele

R. van Velissaropoulos 
P.

1 Victoria-Troncoso

V.

210

372 Vodovozov

A. M,

88 Vrabec

Fr.

342 Vrabec

L

342

Zagora

E.

235 (B) Zer

liana

293 Zingirian

M.

199 Zonis

$\mathrm{S}$.

321 